Supporting Information

\title{
Twisted Bilayer Graphene: A Versatile Fabrication Method And The Detection Of Variable Nanometric Strain Caused By Twist-Angle Disorder
}

\author{
Andreij C. Gadelha, ${ }^{1}$ Douglas A. A. Ohlberg, ${ }^{2}$ Fabiano C. Santana, ${ }^{1}$ Gomes \\ S. N. Eliel,${ }^{3,4}$ Jessica S. Lemos,${ }^{1}$ Vinícius Ornelas, ${ }^{1}$ Daniel Miranda, ${ }^{1}$ \\ Rafael Battistella Nadas, ${ }^{1}$ Kenji Watanabe, ${ }^{5}$ Takashi Taniguchi, ${ }^{5}$ Cassiano \\ Rabelo, ${ }^{6}$ Pedro Paulo de Mello Venezuela, ${ }^{4}$ Gilberto Medeiros-Ribeiro, ${ }^{7}$ \\ Ado Jorio, ${ }^{1,6}$ Luiz Gustavo Cançado, ${ }^{1}$ and Leonardo C. Campos*1 \\ ${ }^{1}$ Physics Department, Universidade Federal de Minas \\ Gerais, Belo Horizonte, MG 31270-901, Brasil. \\ ${ }^{2}$ Microscopy Center, Universidade Federal de Minas \\ Gerais, Belo Horizonte, MG 31270-901, Brasil. \\ ${ }^{3}$ Instituto de Física, Universidade Federal da Bahia, Campus \\ Universitário de Ondina, Salvador - BA, 40170-115 Brasil.
}

${ }^{4}$ Instituto de Fúsica, Universidade Federal Fluminense, Campus da Praia Vermelha Av. Gal. Milton Tavares de Souza, s/no - CEP 24210-346 - Niterói - RJ

${ }^{5}$ National Institute for Materials Science (NIMS), 1-2-1 Sengen, Tsukuba-city, Ibaraki 305-0047, Japan.

${ }^{6}$ Electrical Engineering and Technology Innovation Graduate Programs, Universidade Federal de Minas Gerais, Belo Horizonte, MG 31270-901, Brasil.

${ }^{7}$ Computer Science Department, Universidade Federal de Minas Gerais, Belo Horizonte, MG 31270-901, Brasil. 


\section{Corresponding Author}

Correspondence and requests for materials should be addressed to Leonardo Cristiano Campos - email: lccampos@fisica.ufmg.br

\section{Fabrication of the pyramid stamp (PS)}

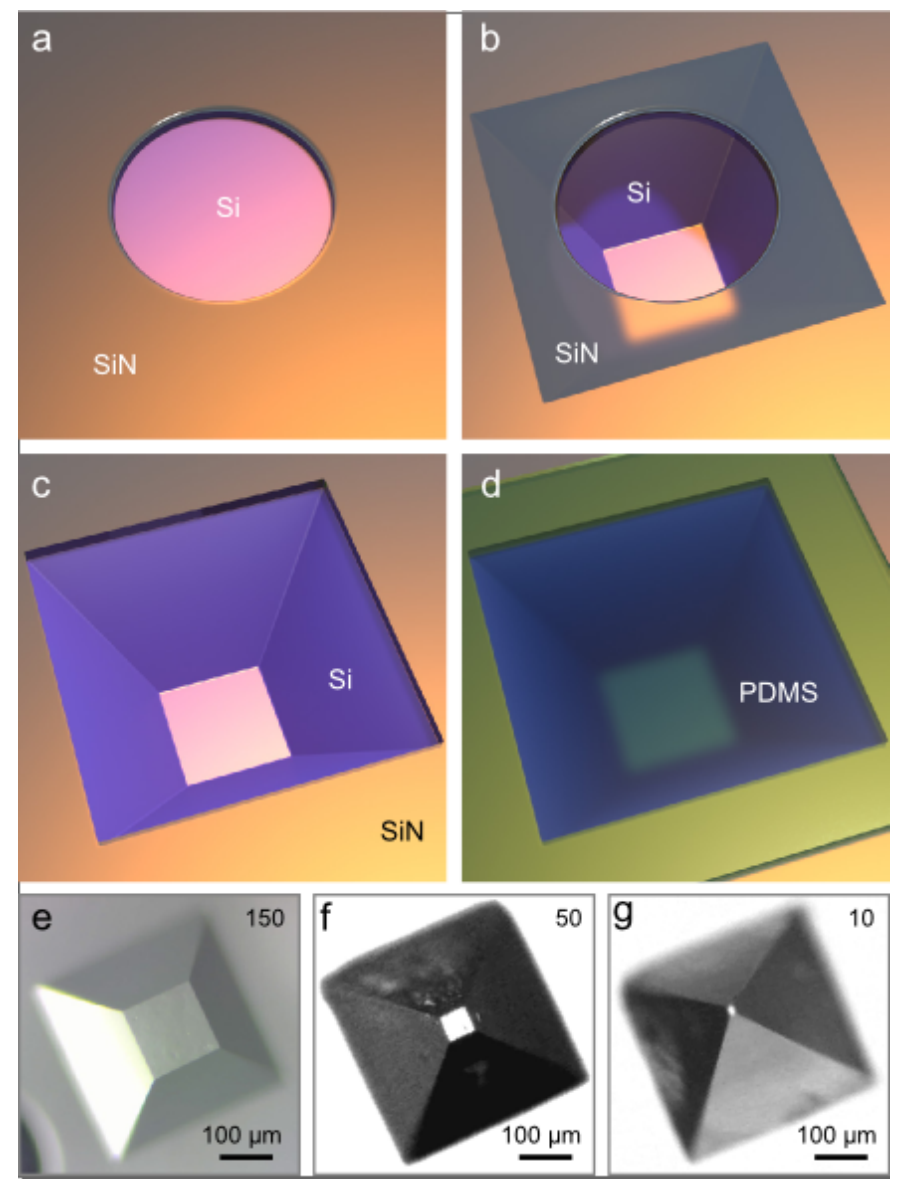

Figure S 1. Fabrication of the PS. a) Hole on a SiN layer covering a Si substrate. b) Etched Si substrate underneath of SiN cover layer. c) Si mold ready for preparing a PS. d) Filling the Si mold with PDMS. e) Optical micrograph of a $150 \mu \mathrm{m}$ wide PS. f) Optical micrograph of a $50 \mu \mathrm{m}$ wide PS. g) Optical micrograph of a $10 \mu \mathrm{m}$ wide PS.

Here we describe the fabrication of the Pyramid Stamp. It is relatively simple and only involves standard clean-room fabrication steps. In the first step, a pyramidal template is created to work as a mold for the PDMS (see Figure S1).The PS mold is composed of a 


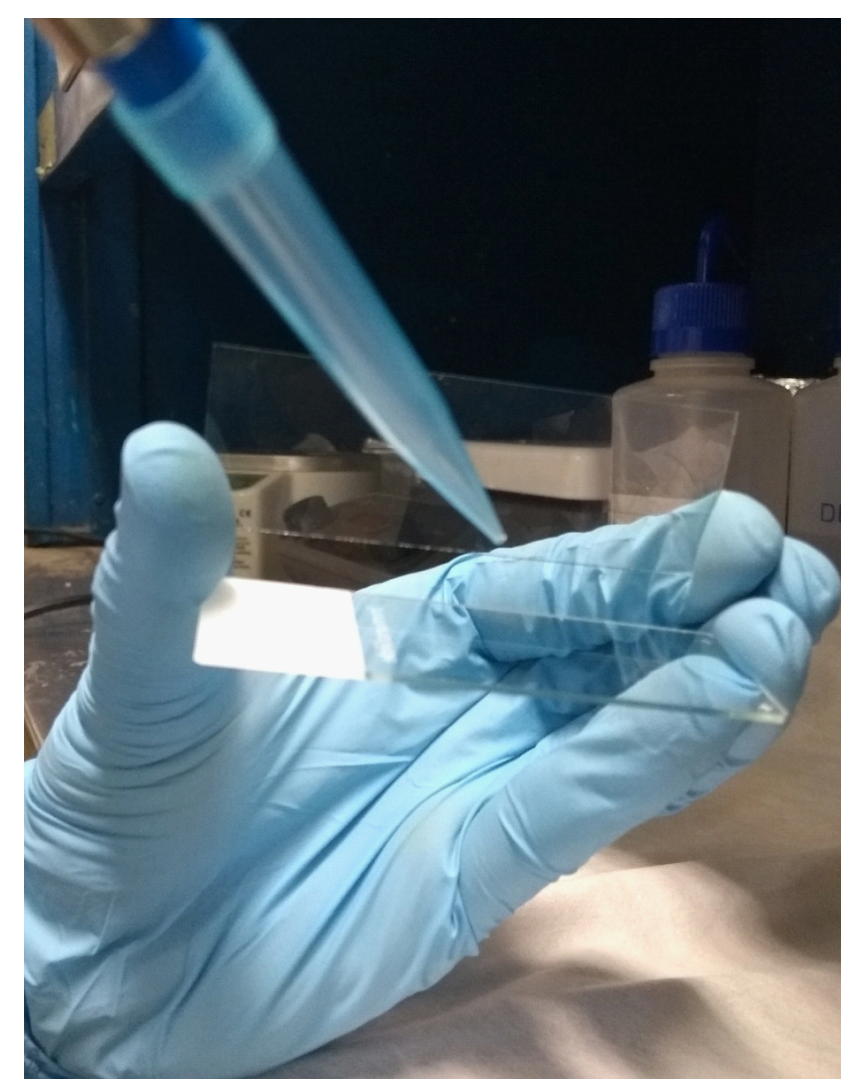

Figure S 2. Preparation of the PC layer.

$\mathrm{Si}(100)$ substrate covered with a $300 \mathrm{~nm}$ thick SiN layer. The Si wafer is covered with a photoresist. We use a Microposit S1813 positive photoresist, which turns to a $1.5 \mu \mathrm{m}$ thick cover layer after spin coat at $4000 \mathrm{RPM}$ for $3 \mathrm{~min}$, followed by a bake out at $T=100{ }^{\circ} \mathrm{C}$ for 2 min. Next, holes are patterned in the photoresist using optical lithography. The best results are $300 \mu \mathrm{m}$ holes separated by $2000 \mu \mathrm{m}$. In a single wafer, an array of holes is fabricated enabling parallel production of PS. The next step is the plasma etching process. Applying a $\mathrm{SF}_{6}+\mathrm{O}_{2}$ plasma for $20 \mathrm{~min}$, all $\mathrm{SiN}$ in the holes is etched away up to the $\mathrm{Si}(100)$, see Figure $\$ 1 \mathrm{a}$. Next step is to perform an anisotropic etching of the silicon using an aqueous solution of potassium hydroxide $(\mathrm{KOH})$ [1-3], see Figure $\$ 1 \mathbf{b}$. For that, the recipe is to insert the wafer in an aqueous $\mathrm{KOH}$ solution ( $45 \%$ in weight at a temperature of $\mathrm{T}=70^{\circ} \mathrm{C}$ and the time is a free parameter. This process etches the silicon preferentially along the $\mathrm{Si}(111)$ direction, forming angles of $\approx 55^{\circ}$ in a pyramidal geometry, see Figure $\mathrm{S} 1 \mathrm{~b}$. The etching rate of $\mathrm{KOH}$ solution over the silicon is $1.5 \mu \mathrm{m} / \mathrm{min}$, and the corrosion time allows the determination of both the height and the width $(w)$ of the semi-pyramidal mold, see 


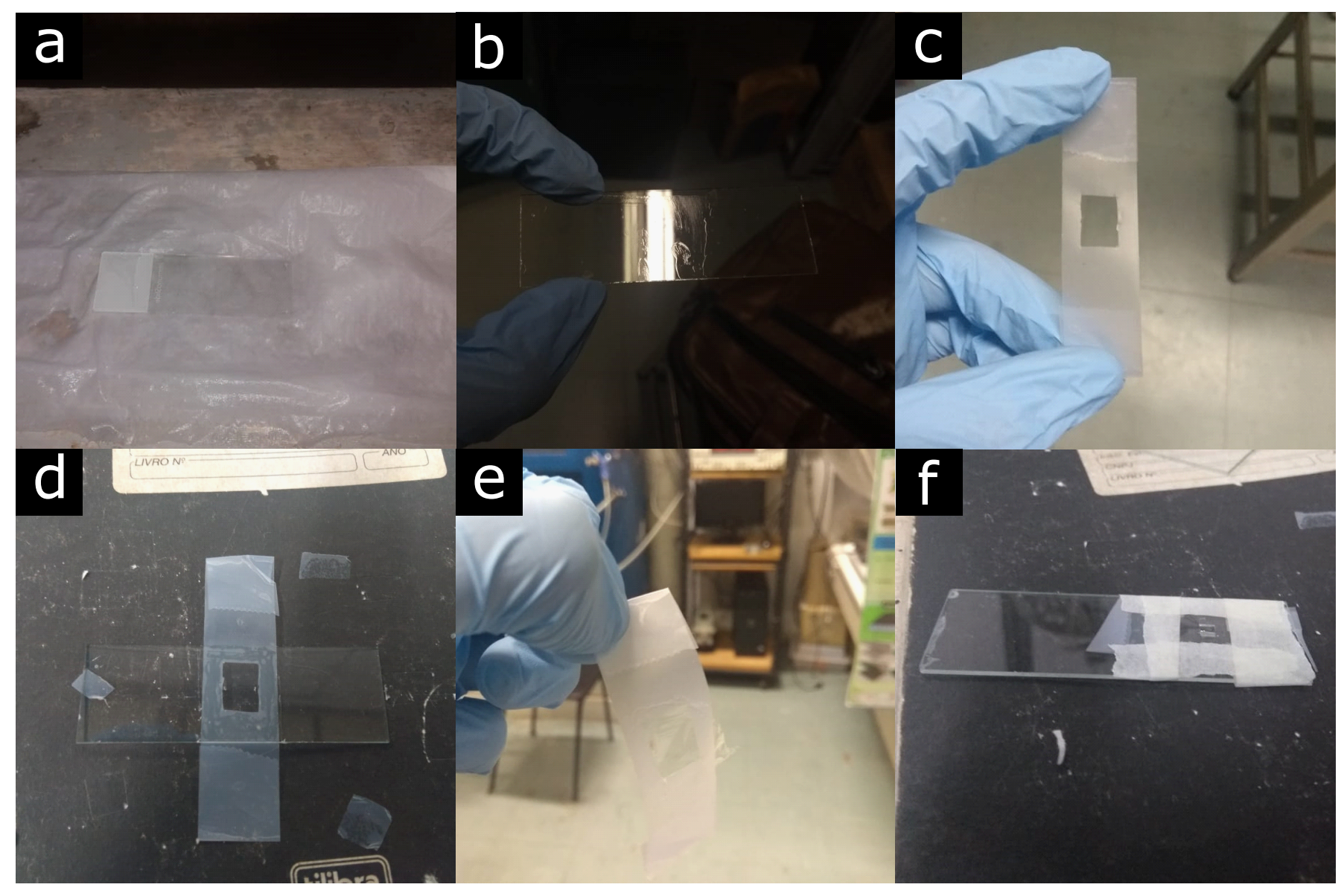

Figure S 3. Preparation of the PC membrane. a) Glass slide covered with the PC layer is placed facing up on top of a humid paper for 15 min. b) PC film is ready covering the glass slide. c) Preparation of a rectangular or square window on a piece of scotch tape. d) Scotch tape is used to pick-up a PC layer. e) Scotch tape is removed from the glass slide removing the PC layer. f) The scotch tape and the PC layer are placed covering the PS on a transparent glass slide.

Figure S1b. After the corrosion, freestanding SiN pieces are observed covering the template, and they are easily removed after sonication in deionized water, see Figure S1 c. Molds with different sizes are achieved changing the etching time. For instance, a semi-pyramidal mold $150 \mu \mathrm{m}$ wide is obtained after etching the Si wafer for 2 hs. After etching it for 6 hs the plateaus is $50 \mu \mathrm{m}$ wide, and etching it for $7 \mathrm{hs}$ the final plateau is $10 \mu \mathrm{m}$ wide, see Figures S1e-g. Different plateau sizes can be used for different purposes. In our work, we were happy with $150 \mu \mathrm{m}$ wide plateaus which provided very good alignments as well as graphene visualization. Finally, after having the etched Si mold, the next step is to fill the mold with PDMS, see Figure S1d, and after curing, the PS is ready for use. To prepare the PDMS, 
we use the SYLGARD(R) 184 SILICONE ELASTOMER KIT with the proportion of 1:10 between the curing agent and the base. After filling the mold with PDMS, it is leaved in a vacuum desiccator for roughly five minutes to remove bubbles. After that, to reduce the PDMS curing time, it is heated in a hot plate at $T=100^{\circ} \mathrm{C}$ for ten minutes. The PDMS is then removed from the mold. To do that, it is cut using a razor blade in parts containing the PS, and we pick it up with tweezers. At the very end of the process, it is deposited on a glass slide. No glue is necessary, because the PDMS sticks naturally to the glass slide.

Next, we prepare a polycarbonate (PC) solution using solid PC (Poly Bispheol A Carbonate) bought from sigma-aldrich and diluted in chloroform at a $10 \%$ mass concentration. Then, we drop $500 \mu \mathrm{l}$ of the solution on a clean glass slide, like seen in Figure $\$ 2$, and cover it with other clean slide, pressing the solution between the two slides and sliding them laterally. Next, we heat the slides up to $120^{\circ} \mathrm{C}$ for 15 minutes. After this process, we leave the membrane, with the PC layer facing up, on a humid paper, see Figure S3(a). Finally, the $\mathrm{PC}$ is ready to be used Figure $\mathrm{S} 3(\mathrm{~b})$. Next, by making a square window on a piece of scotch tape (bought from 3M) Figure $\mathrm{S} 3(\mathrm{c})$, we pick-up the PC layer Figure $\mathrm{S} 3(\mathrm{~d})$, so the window on Figure S3(c) now contain a freestanding PC layer Figure S3(e). Then, we deposit the PC on the PS Figure $\mathrm{S} 3(\mathrm{f})$, and we attach it with masking tape.

\section{Transferring TBG from $\mathrm{SiO}_{2}$ to mica/Au substrates}

As it is schematically shown in Figure $\mathbf{S} \mathbf{4}$, the pick-up procedure consists of putting the PS in contact with the TBG on $\mathrm{SiO}_{2}$ substrate at $T=70^{\circ} \mathrm{C}$, raising the temperature to $T=80^{\circ} \mathrm{C}$, cooling it down to $T=70^{\circ} \mathrm{C}$ and then separating the PS from the substrate. The TBG sticks on the PS, and it is removed from the $\mathrm{SiO}_{2}$ substrate in this step. In the sequence, it was then put in contact with the conducting mica/Au substrate required for STM measurements, after which the temperature was raised to $T=180^{\circ} \mathrm{C}$ to transfer the TBG with the polycarbonate capping layer of the PS. Finally, the PC layer is removed in chloroform and isopropyl alcohol bath. Again, the recipe is $15 \mathrm{~min}$ at $40^{\circ} \mathrm{C}$ in chloroform and $1 \mathrm{~min}$ in isopropyl alcohol at room temperature. In Figure $\mathrm{S} 4 \mathbf{b}$, we present an optical micrograph of the TBG on the $\mathrm{SiO}_{2}$ substrate before the transferring and in Figure S4c we present a optical picture after transfer it to a mica/Au substrate. Substantial regions are preserved and approximately the same twist-angle has been verified by the sMIM and STM 


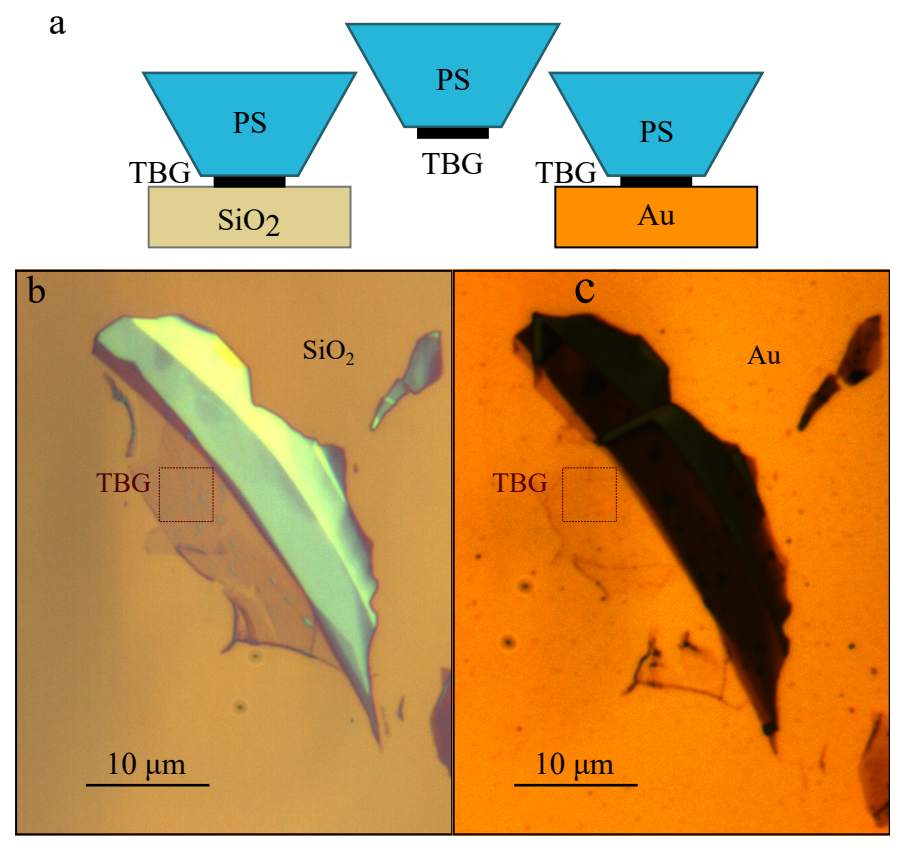

Figure S 4. Transferring TBG from $\mathrm{SiO}_{2}$ to mica/Au substrates. a) From the left to the right: a schematic cartoon of the transferring procedure used to transfer the TBG from $\mathrm{SiO}_{2}$ substrate to a mica/Au substrate. b) Optical picture of a $\mathrm{TBG}$ on $\mathrm{SiO}_{2}$ substrate. c) Optical picture of the same TBG transferred to a mica/Au substrate.

data presented in the main text.

A relevant result compares the topographies of the samples on $\mathrm{SiO}_{2}$ and mica/Au, particularly the rearrangement of bubbles and wrinkles. Figure \$5 shows topography images (height) acquired from the AFM and STM equipment. In the top panel in Figure $\$ 5$, we present large AFM topography images. On the left side, we depict an AFM image captured before the transferring, and on the right side, we present a mosaic of topography images acquired after transferring the TBG to a mica/Au substrate. In the STM equipment, the scan size is limited, and it is not possible to scan over the whole sample once. Right below, Figures S5(a)-(b) depict AFM data captured from the green squared highlighted in the top panel. The transferring process changed the wrinkle and bubble patterns in many regions of the sample. Overall, the main similarities between the AFM (performed before) and STM (performed after transferring) were in the multilayer regions and the edges of the bilayer regions. Only in a few areas of the twisted bilayer graphene, we could find portions of intact transferred wrinkles. Comparing the AFM image acquired before and after the transferring, one can see that the transferred sample is not identical to what it was before. However, 


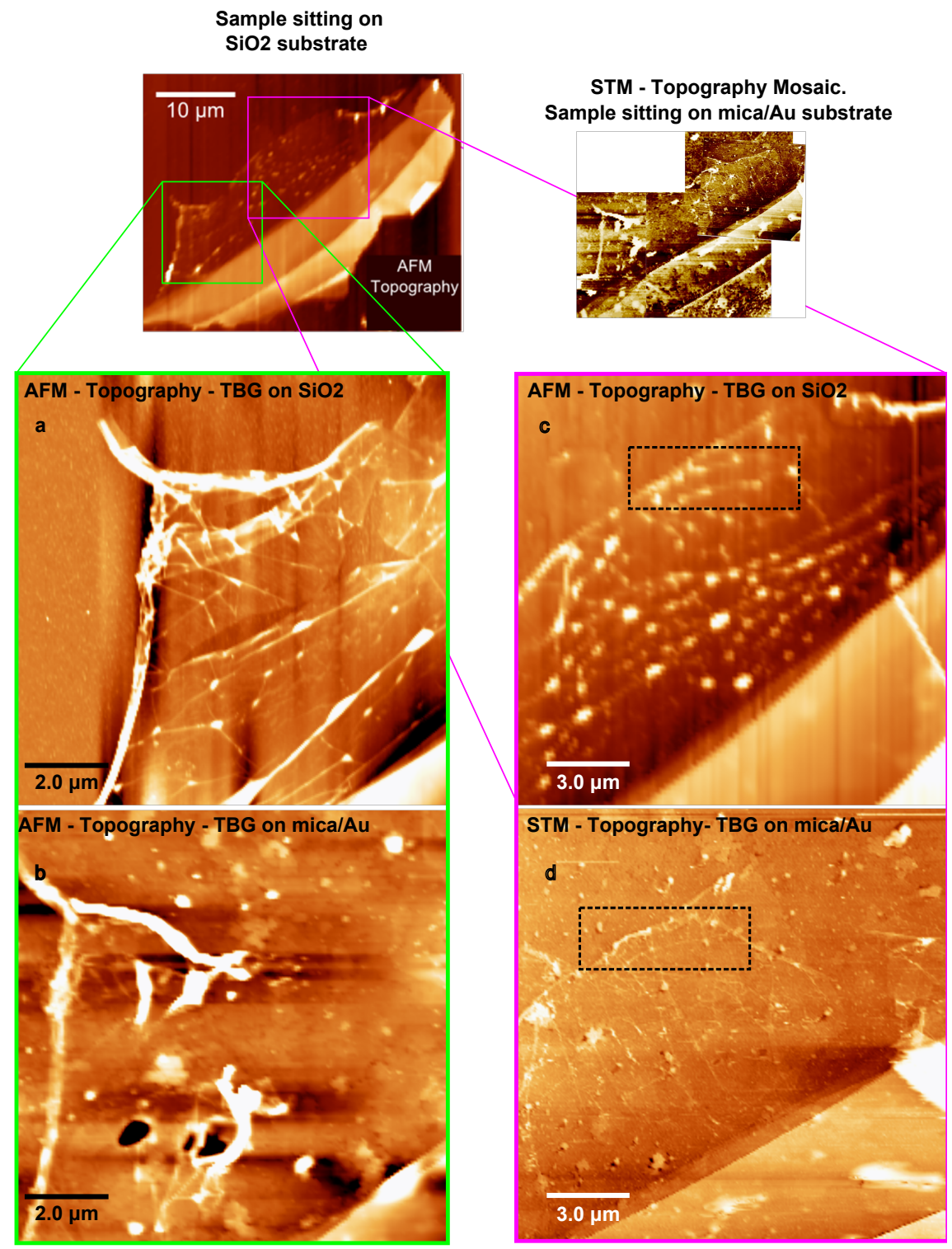

Figure S 5. AFMs topography scanned before and after transferring the TBG from $\mathrm{SiO}_{2}$ substrate to mica/Au substrate. a) AFM topography image recorded from the area highlighted by the green square on the top panel. TBG sitting on $\mathrm{SiO}_{2}$ substrate - before transferring the TBG. b) AFM topography image recorded from the area highlighted by the green square on the top panel. TBG sitting on mica/Au substrate - after transferring the TBG. c) AFM topography image recorded from the area highlighted by the pink square on the top panel. TBG sitting on $\mathrm{SiO}_{2}$ substrate before transferring the TBG. d) AFM topography image recorded from the area highlighted by the pink square on the top panel. TBG sitting on mica/Au substrate - after transferring the TBG. 
some wrinkles can be recognized after the transferring. The same is valid for other areas like the one showed in Figures $\$ 5$ (c)-(d). The wrinkles are not the same, except for a few observed on the top central region highlighted by the black rectangle.

\section{Nano-Raman - Tip-enhanced Raman spectroscopy (TERS)}

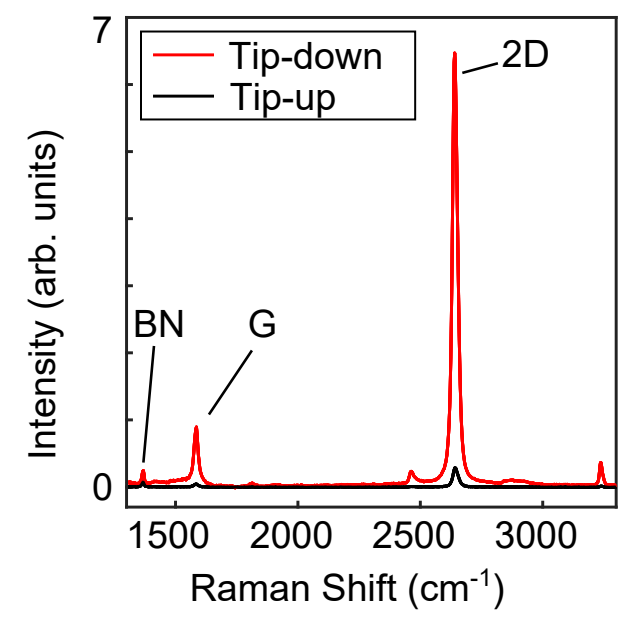

Figure S 6. Raman spectra from a graphene flake, recorded with a plasmonic tip [4] in contact (red) and retracted (black).

Tip-enhanced Raman spectroscopy [5, 6] enables strong enhancement of the Raman signal, with spatial resolution beyond the diffraction limit. The spatial resolution is defined by the area under the tip, which is $\approx 10 \mathrm{~nm}$ in our experiments. Figure $\mathrm{s} 6$ presents Raman spectra of a graphene piece recorded with a plasmon tunable pyramid tip (PTTP) [4 in contact (tip-down, red line) and retracted (tip-up, black line). The peak from hBN, as well as the $\mathrm{G}$ and $2 \mathrm{D}$ bands from graphene are highlighted. In this experiment, the nano-Raman intensity was enhanced by 20 -fold if compared with the micro-Raman signal.

\section{sMIM characterization of a homogeneous and a heterogeneous twisted bilayer graphene on a hBN crystal}

In Figure 5 of the main text, we show that nano-Raman spectroscopy is a powerful toll able to characterize local strain and identify regions under non homogeneous strain. Here, in Figure S7, we present a broader investigation of the two TBG regions presented in Figure 5 
of the main text. In the inset of Figure $\$ 7 \mathbf{a}$, we show a Micro-Raman hyperspectral imaging where the blue shade regions correspond to the TBG areas. In the central-lower region, we draw a red rectangle, a red circle, and a red triangle at the locals where the sMIM data were captured, as presented in Figure S7 $\mathbf{a}-\mathbf{c}$, respectively. Similarly, in the central-right region, we added a green rectangle at the region where the sMIM data presented in Figure S7 d were measured. Similarly to what is presented, in all sMIM data we show the component of the reflected microwave signal which is a function of the local conductivity of the sample.

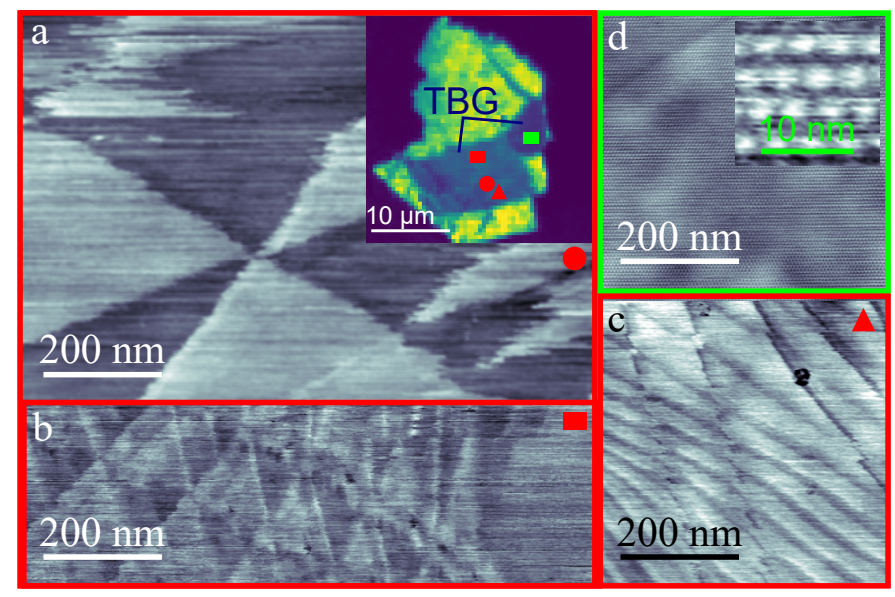

Figure S 7. Characterization of the TBG sample presented in Figure 5 of the main text by near field scanning microwave microscopy (sMIM). a) sMIM data acquired from the red rectangular region depicted in the inset Figure S7a. In the inset, we present the same Micro-Raman hyperspectral image presented in Figure 5a from the main text showing the TBG regions. b) sMIM data acquired from region highlighted by the red circle presented in the inset Figure $\$ 77$. c) sMIM data acquired from region highlighted by the red triangle presented in the inset Figure S7a. d) sMIM data acquired from the green rectangular region depicted in the inset Figure S7a. In the Inset, we present a zoom-in in the region showing a regular Moiré pattern.

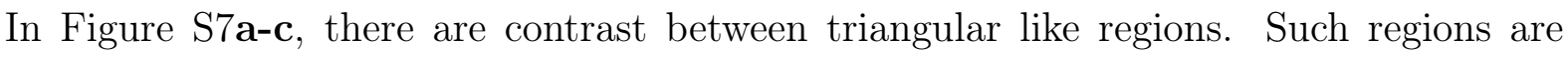
the $\mathrm{AB}$ or BA Bernal stacking regions typical from TBG samples with small twist-angle $\left(\theta<1.1^{\circ}\right)$, showing crystal lattice reconstruction. [7] Notoriously, Figure S7a-c exhibit Bernal stacking regions changing in shape and in size over the whole central-lower region of the TBG, what strongly suggest that the TBG is under variable strain. The data presented in Figure 5c-e are captured from the same region marked by the red rectangle in the inset 
of Figure $\$ 7 \mathbf{a}$, and the size of the Bernal staking regions are similar to the size of the region measured by TERs. Therefore, the data presented in Figure $5 \mathbf{c}-\mathbf{e}$ very likely confirms that the TBG is under variable strain in this region. On the other hand, nano-Raman data presented in Figure $5 \mathbf{f}-\mathbf{h}$ are acquired from the central-right region, where we draw a green rectangle. The whole central-right region is considerable more homogeneous than the central-lower region, and the typical sMIM data is presented in Figure $\mathrm{S} 7 \mathrm{~d}$. In the inset of Figure $\mathrm{S} 7 \mathrm{~d}$ a zoom in the sMIM data is presented revelling a Moiré superlattice with very regular pattern. 


\section{References}

[1] Ferrari, G.; Oliveira, A. B.; Silvestre, I.; Matos, M. J. S.; Batista, R. J. C.; Fernandes, T. F. D.; Meireles, L. M.; Eliel, G. S. N.; Chacham, H.; Neves, B. R. A.; Lacerda, R. G. Apparent Softening of Wet Graphene Membranes on a Microfluidic Platform ACS Nano 2018, 12, 43124320.

[2] Meireles, L. M.; Eliel, G. S. N.; Ferrari, G. A.; Neves, P. A. A.; Gadelha, A. C.; Silvestre, I.; Taniguchi, T.; Watanabe, K.; Chacham, H.; Neves, B. R. A.; Campos, L. C.; Lacerda, R. G. Graphene Electromechanical Water Sensor: The Wetristor Advanced Electronic Materials 2020, 6, 1901167.

[3] Meireles, L. M.; Barcelos, I. D.; Ferrari, G. A.; Neves, P. A. A. A.; Freitas, R. O.; Lacerda, R. G. Synchrotron infrared nanospectroscopy on a graphene chip Lab Chip 2019, 19, 3678-3684.

[4] Vasconcelos, T. L.; Archanjo, B.; Oliveira, B. S.; Valaski, R.; Cordeiro, R. C.; Medeiros, H. G.; Rabelo, C.; Ribeiro, A.; Ercius, P.; Achete, C. A.; Jorio, A.; Cançado, L. G. Plasmon-Tunable Tip Pyramids: Monopole Nanoantennas for Near-Field Scanning Optical Microscopy. Advanced Optical Materials 2018, 6(20), 1800528.

[5] Beams, R.; Cançado, L. G.; Jorio, A.; Vamivakas, N.; Novotny, L. Tip-enhanced Raman mapping of local strain in graphene. Nanotechnology 2015, 26, 175702.

[6] Rabelo, C.; Miranda, H.; Vasconcelos, T. L.; Cançado, L. G.; Jorio, A. Tip-enhanced Raman Spectroscopy of Graphene. In 2019 4th International Symposium on Instrumentation Systems, Circuits and Transducers (INSCIT) - IEEE 2019, 1-6.

[7] Yoo, H.; Engelke, R.; Carr, S.; Fang, S.; Zhang, K.; Cazeaux, P.; Sung, S. H.; Hovden, R.; Tsen, A. W.; Taniguchi, T.; Watanabe, K.; Yi, G.; Kim, M.; Luskin, M.; Tadmor, E. B.; Kaxiras, E.; Kim, P. Atomic and electronic reconstruction at the van der waals interface in twisted bilayer graphene. Nature materials 2019, 18(5), 448-453. 